\title{
Programmed Control Scheme For Air Contamination And Hazards Revelation In Vehicles
}

\author{
1. D.KAVYA,PG Student,2.PANEM CHARANARUR,MIEEE,Assistant Professor \\ Department of ECE,CBTVIT,Hyderabad
}

\begin{abstract}
Now a day's population has been increased rapidly. Along with the population pollution also increases. In earlier cases a common man vehicle is by cycle. But now a day the cycle was replaced with motor cars and bikes. If the vehicles are not maintained in proper manner pollution get increases. It might be because of people negligence or government negligence or entire system negligence. In order to avoid this problem it could be great integrated with automated control system for Air pollution And Disaster detection in vehicles. Here the pollution is not avoided completely. But it can be controlled. The main aim of it uses semi conductor sensors at emission outlets of vehicles. It also detects the level of pollutants and indicates that with a level of meter. It uses a threshold level. When the pollution level shootsbeyond the threshold level the motor speed will be reduced after certain period of time. This will detect the level of pollution and indicating it to driver. During this time period the GPS will starts locating the nearest stations. After the timer runs out. The fuel supplied to engine will be cut off and vehicle will be stopped. The Existing system consisting of Which is used to detect the pollution level and give signal to the fuel injector through Microcontroller which will cut the fuel to the engine and trace the maintenance centre in that area through the GPS. The proposed system consisting of the microcontroller is placed with the LPC2148 processor to improve the efficiency. An additional device is going to added for accident information purpose i.e. an alert message is send to the relation phone using GSM.

Sensors are used in everyday objects such as touch-sensitive elevator buttons (tactile sensor) and lamps which dim or brighten by touching the base. There are also innumerable applications for sensors of which most people are never aware. These happen on most factors if the driver is drowsy or if he is alcoholic. Driver drowsiness is recognized as an important factor in the vehicle accidents. It was demonstrated that driving performance deteriorates with increased drowsiness with resulting crashes constituting more than $20 \%$ of all vehicle accidents. But the life lost once cannot be re-winded. Advanced technology offers some hope avoid these up to some extent. MEMS easily grab the information from vehicle, if any change occurs above threshold voltage immediately it sends signal to LPC2148 processor. Immediately it decodes GPS values and sends to consult person using GSM.
\end{abstract}

Index Terms-LPC2148,MEMS,GPS,GSM,Tactile Sensor.

\section{INTRODUCTION}

An Embedded System is a combination of computer software and hardware, A system is a way of working, organizing or or doing one or many tasks according to fixed plan. Program or set of rules. And it can perhaps other parts or additional mechanical, \& designed to perform a specific function. A good example is of an embedded system is the microwave oven. Almost every household has one, and tens of millions of them are used every day. This is in direct contrast to the personal computer in the family room. It is to comprised of computer software and hardware and mechanical components the example of this components are disk drivers. However, a personal computer is not designed to perform a specific function rather; it is able to do many different things. Many people use the term generalpurpose computer to make this distinction clear. As shipped, a general-purpose computer is a blank slate; the manufacturer does not know what the customer will do wish it. One customer may use it for a network file server another may use it exclusively for playing games.

Wireless technologies Advances in mobile communications are paving way for many interesting applications using embedded systems. The mobile phone is one of the marvels of the last decade of the $20^{\text {'th }}$ century. It is a very powerful embedded system that provides voice communication while are on the move. The Personal Digital Assistants and the palmtops can now be used to access multimedia services over the Internet.
Mobile communication infrastructure such as base station controllers, mobile switching centers are also powerful embedded systems. Examples of wireless technologies are mobile phones and wireless LAN systems. For mobile phones it supports full duplex communication. While the GSM mobiles uses special identity. CDMA mobiles do not require. LANs are enables communication between two or more systems without using wired connection. It supports communication only for limited surrounding area.

The main aim of it uses semiconductor sensors at emission outlets of vehicles. It also detects the level of pollutants and indicates that with a level of meter. It uses a threshold level. When the pollution level shoots beyond the threshold level the motor speed will be reduced after certain period of time. This will detect the level of pollution and indicating it to driver. During this time period the GPS will starts locating the nearest stations. After the timer runs out. The fuel supplied to engine will be cut off and vehicle will be stopped. The Existing system consisting of Which is used to detect the pollution level and give signal to the fuel injector through Microcontroller which will cut the fuel to the engine and trace the maintenance center in that area through the GPS. The proposed system consisting of the microcontroller is placed with the LPC2148 processor to improve the efficiency. An additional device is going to added for accident information purpose i.e. an alert message is send to the relation phone using GSM. Sensors are used in everyday objects such as touch-sensitive elevator buttons (tactile sensor) and lamps which dim or brighten by 
touching the base. There are also innumerable applications for sensors of which most people are never aware. Applications include cars, machines, aerospace, medicine, manufacturing and robotics. A sensor is a device which receives and responds to a signal when touched. A sensor's sensitivity indicates how much the sensor's output changes when the measured quantity changes. Vehicle accidents are most common if the driving is inadequate.

These happen on most factors if the driver is drowsy or if he is alcoholic. Driver drowsiness is recognized as an important factor in the vehicle accidents. It was demonstrated that driving performance deteriorates with increased drowsiness with resulting crashes constituting more than $20 \%$ of all vehicle accidents. But the life lost once cannot be re-winded. Advanced technology offers some hope avoid these up to some extent. MEMS easily grab the information from vehicle, if any change occurs above threshold voltage immediately it sends signal to LPC2148 processor. Immediately it decodes GPS values and sends to consult person using GSM.

In this paper describe the following areas, describes about the introduction to the whole project. describes about the hardware requirements regarding the project, describes about the software tools used in the project, describes about the Hardware implementation of project, describes Software development regarding to the project, describes the results generated by using kiel and flash magic, describes the conclusion, future scope, advantages and disadvantages of the work.

\section{DESCRIPTION OF HARDWARE REQUIREMENTS}

\subsection{LPC2148}

The LPC2148 microcontrollers are based on a 32/16 bit ARM7TDMI-S CPU with real-time emulation and embedded trace support, that combines the microcontroller with embedded high speed flash memory ranging from $32 \mathrm{kB}$ to $512 \mathrm{kB}$. A 128-bit wide memory interface and unique accelerator architecture enable 32- bit code execution at the maximum clock rate. For critical code size applications, the alternative 16-bit Thumb mode reduces code by more than $30 \%$ with minimal performance penalty. Due to their tiny size and low power consumption, LPC2141/2/4/6/8 are ideal for applications where miniaturization is a key requirement, such as access control and point-of-sale. A blend of serial communications interfaces ranging from a USB 2.0 Full Speed device, multiple UARTS, SPI, SSP to I2Cs and onchip SRAM of $8 \mathrm{kB}$ up to $40 \mathrm{kB}$, make these devices very well suited for communication gateways and protocol converters, soft modems, voice recognition and low end imaging, providing both large buffer size and high processing power. Various 32-bit timers, single or dual 10-bit ADC(s), 10-bit DAC, PWM channels and 45 fast GPIO lines.

The LPC2148 consists of an ARM7TDMI-S CPU with emulation support, the ARM7 Local Bus for interface to on-chip memory controllers, the AMBA
Advanced High-performance Bus (AHB) for interface to the interrupt controller, and the VLSI Peripheral Bus (VPB, a compatible superset of ARM's AMBA Advanced Peripheral Bus) for connection to on-chip peripheral functions. The connection of on-chip peripherals to device pins is controlled by a Pin Connect Block.

Applications of Industrial control, Medical systems, Communication gateway, Embedded soft modem, General purpose applications.

\subsection{MEMS Sensor}

The abbreviation of the MEMS is a micro-electro mechanical chip which is used to recognize the vibrations or movements from any device or from human. MEMS mainly used for detecting the gestures of the hands of human. This is also used to detect the earth quakes etc. the output of the MEMS is in digital form with $3.6 \mathrm{~V}$. It is a three-axis accelerometer used very low power. Sensor has an interrupt pin which is named as INT used to detect the gestures, orientation of any device, changing of the data. The device is housed in a small $3 \mathrm{~mm} \times 3 \mathrm{~mm} \times 0.9 \mathrm{~mm}$ DFN package.

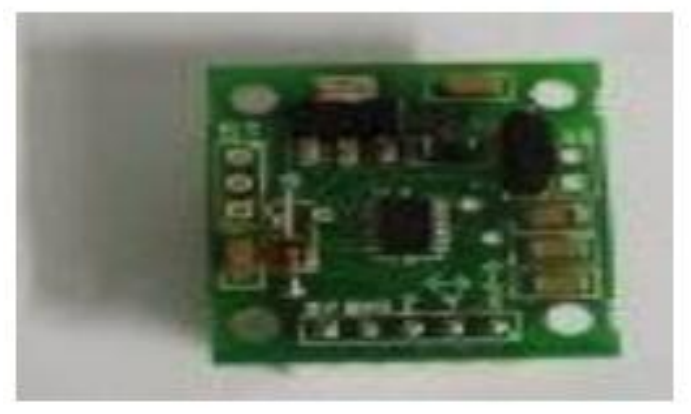

Figure 2.1 MEMS sensor module

Many elements are integrated to build the Micro-ElectroMechanical Systems (MEMS) which are named as actuators, mechanical components, sensors, and electronics. Micro fabrication technology is used to pack all these elements on a silicon substrate. CMOS, BICMOS or BIPOLAR fabrication method are used as Integrated circuits. By using hands and arms of the human, MEMS can be operated to control the processors or micro controllers. These sensors can sense many types of environment changes like optical, thermal, chemical, biological, mechanical and magnetic effects. The detection made by the sensors provide some information to the actuators which are processed and decisions are given by the human to the controller which are as filtering, pumping, regulating and positioning etc. For desired output the environment is controlled through these sensors.

The Beginners guide to MEMS Processing To know about the MEMS technology there are many methods and tools which are used to create structure with small dimensions in a scale of micro-meter. Fromm IC (Integrated circuits) technology itself this method is adopted. For example, in maximum method silicon wafers are only used. Like IC's only structures are made with thin films. Communications The advanced MEMS technology leads to beneficial in the high frequency circuits. The communication circuit's 
performance increases due to the integration of these electronic components sop that the power consumption, area of the circuit and cost will be decreased. In many microwave circuits mechanical switch is the main key component. The mechanical switch is very higher than other components when compared.

Accelerometers In automobiles like cars, trucks the MEMS sensors are mainly used to detect the accident occurrence through their accelerometers which are inserted in the Air-bag system. Many of bulky accelerometers are used for the conventional approach which is made by the discrete components placed in-front of the car to detect the vibrations through accidents. MEMS and Nanotechnology has made it possible to integrate the accelerometer and electronics onto a single silicon chip at a cost between $\$ 5$ to $\$ 10$.

\subsection{GPS (Global positioning system):}

To know the person in any place user can use this GPS which is abbreviated as Global positioning system. Through this system user can know navigation, positioning, longitude and latitude positions in the entire world which is freely available to all. The time and location are provided by the GPS receiver. In day or night, any person can know about any other person where he located at what time he reached and anywhere in the world.

\subsection{GSM Modem}

Definitions The words, -Mobile Station (MS) or -Mobile Equipment (ME) are used for mobile terminals Supporting GSM services. A call from a GSM mobile station to the PSTN is called a -mobile originated call (MOC) or -Outgoing call, and a call from Value Description 0 Fix not available or invalid 1 GPS SPS Mode, fix valid 2 Differential GPS, SPS Mode, fix valid 3-5 Not supported 6 Dead Reckoning Mode.

\section{Hardware Implementation}

Description The block diagram consisting of input section ,controller block, output section. The input section consisting of smoke sensor, Mems sensor, GPS, GSM. Here the controller block is LPC2148. The output section consisting of Relay, DC motor, LED and GSM. In the block diagram GSM will be act as a both input as well as output. smoke sensor is widely used in gas detecting equipments. Mems sensor is used for accident indication purpose.GSM will sends a message to the initialized mobile number. GPS will provide its latitude position, longitude position and time. LED is used for indication purpose.

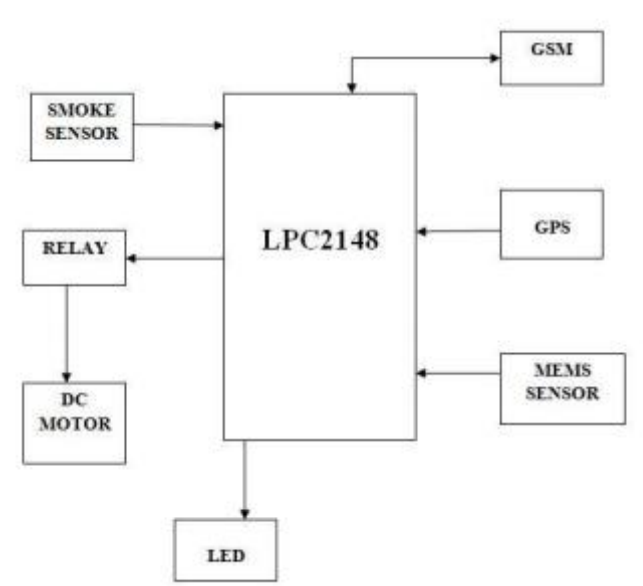

Figure 3.1 Block diagram representation 3.1 Circuit Diagram And Description
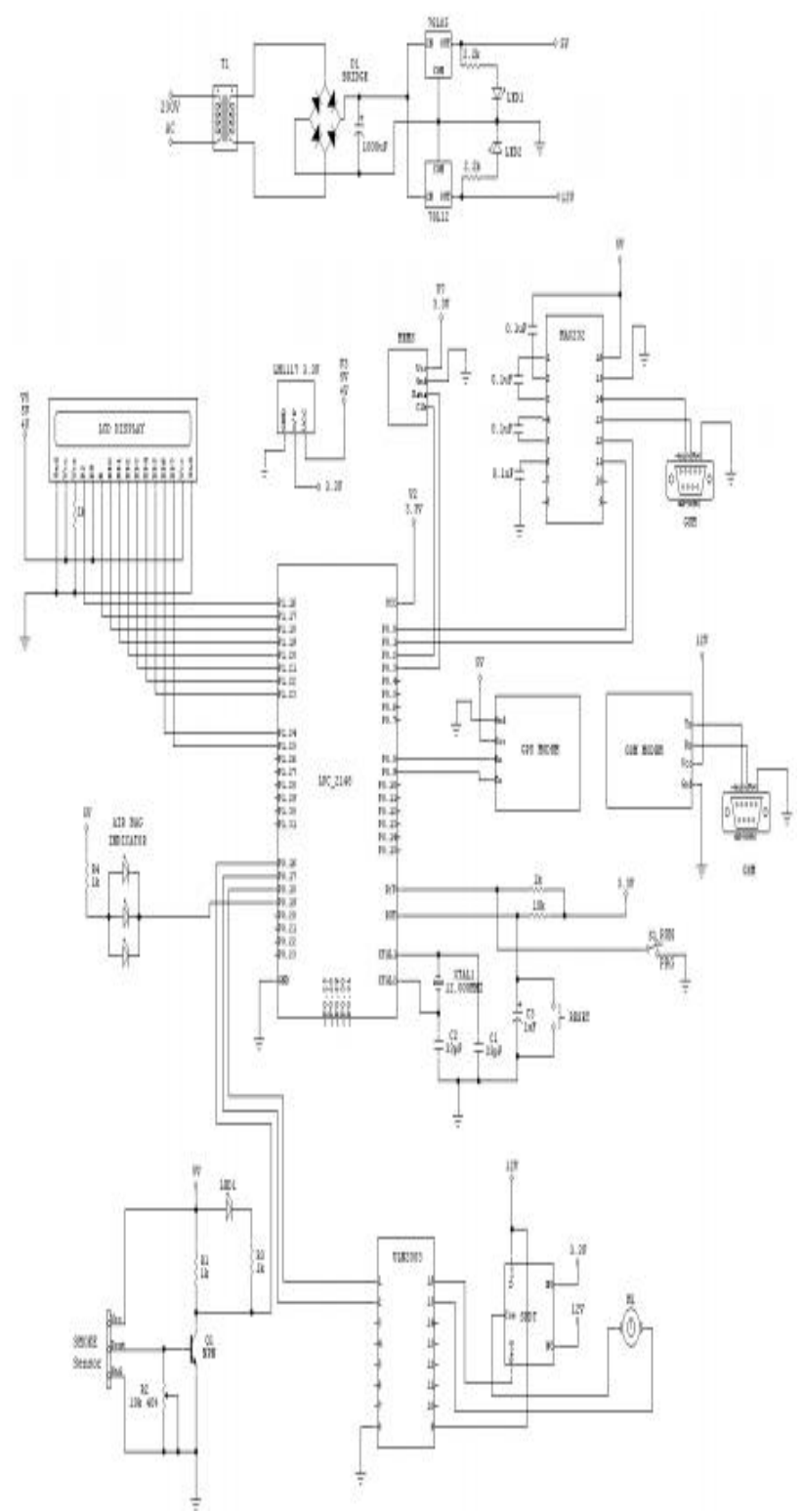

Figure 3.2 Circuit diagram representation 
Description Hardware implementation is divided into three categories.

1 .Input section

2. Controller block

3. Output section.

Hardware implementation deals in drawing the schematic according to the application, testing the schematic design over the breadboard using the various IC's to find if the design meets the objective, carrying out the PCB layout of the schematic tested on breadboard, finally preparing the board and testing the designed hardware. The regulated power supply is shown in circuit diagram. The power supply supplies the power to the all related components. By using the step down transformer in order to decrease the voltage from $230 \mathrm{v}$ AC to $12 \mathrm{v}$ AC. The rectifier used to convert A.C to D.C.by compare all rectifiers ripple factor is less in bridge rectifier. In order to remove pulsating D.C have to use filter capacitor. The regulators are used to provide constant power supply. Lpc2148 is supplied with $3.3 \mathrm{v}$ and for remaining sensor $5 \mathrm{v}$ is supplied from the power supply. The input section consisting of smoke sensor, MEMS sensor, GPS, GSM.The controller block consisting of LPC2148 and

The output section consisting of relay, DC motor, LED, GSM. Smoke sensor The smoke sensor uses a MQ-2 gas sensor. It is a simple drive circuit and used for high sensitivity and fast response of a system. Mainly this sensor is used for detecting methane, alcohol, hydrogen and smoke. MEMS sensor This is the sensor which recognizes the gestures of the hand automatically and any vibrations in the earth or in automobile like cars, trucks etc. So in this project to detect the accident with in time MEMS sensor is used. When the disaster or accident occurred car has some movements and vibrations from the internal parts which can be easily recognized by this sensor. Then it immediately send this signal to GSM, a warning message is send to related mobile number without latency.

GPS Receive data from satellite to LPC2148 Primer Board by using GPS module through UART0. The serial data is taken from the GPS module through MAX232 into the SBUF register of LPC2148 microcontroller. The serial data from the GPS receiver is taken by using the Serial Interrupt of the controller. This is the main device used in this project, when accident has been occurred to vehicle it immediately forwards a message to the mobile number using GSM. In that message have the information about the accident and the area where the vehicle and at what time it has been occurred. All this information is decoded by the GPS.

GSM This device plays vital role in this project; it is that much important because it has to send message in time when the pollution has been increased as well accident has been occurred. Here GSM will act as both input and output. After that have to insert a sim in GSM modem. An adaptor is connected to the regulated power supply is shown into GSM modem. After that have to heated smoke sensor, then GSM modem has to display give missed call, then only have to make a call to the inserted sim. The inserted sim will automatically reject the call and it will send a message i.e;CO2 is occurred. When heat smoke sensor, if the pollution is more the motor speed will be reduced at $(30 \%$ to $35 \%)$. In MEMS sensor if put it in any angle, it will send a message i.e; accident has been occurred at $60 \%$ to $65 \%$. When accident has been occurred LED will automatically ON. With the help of MEMS sensor the GPS will decodes its value and sends a message to the particular mobile number $\mathrm{i}$;e is accident has been occurred.

\section{IV.Software Development}

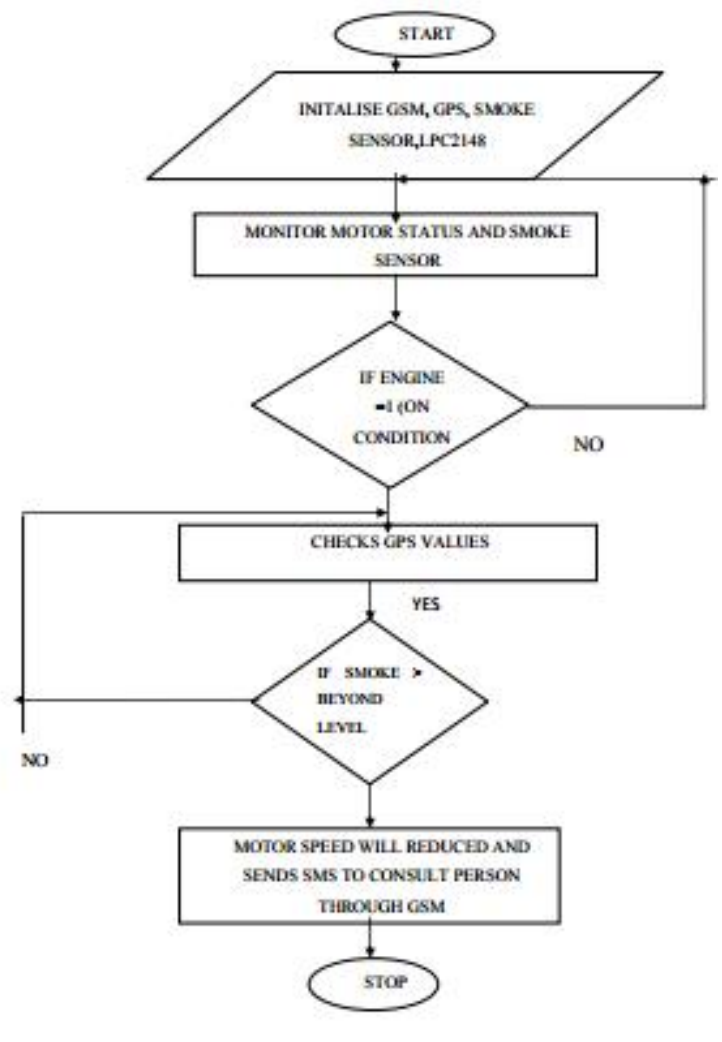

\subsection{Explanation}

The flow graph is shown in the above diagram. The prototype is started by supplying the power. By using the LPC2148 GSM, GPS, smoke sensor are initialized as it is programmed. The status of the smoke sensor is observed; when it crosses the limit automatically motor will reduces its speed. Then the processor checks the engine value if it is not one then again it initializes the smoke sensor, if it is yesthen it checks the GPS value. If smoke sensor exceeds the value, processor will control speed of the motor otherwise it again check the GPS value.

\subsection{Introduction to KIEL}

A compiler for a high level language helps to reduce production time. To program the LPC2148 microcontroller the Keil $\mu \mathrm{v} 4$ is used. The programming is done in the embedded $\mathrm{C}$ language or Assembly language. Keil $\mu v 4$ is a suite of executable, open source software development tools for the microcontrollers hosted on the Windows platform. One of the difficulties of programming microcontrollers is the limited amount of resources the programmer has to deal with. In personal computers resources such as RAM and processing speed are basically limitless when compared to microcontrollers. In contrast, the code on microcontrollers should be as low on resources as possible. 


\subsection{Steps To Implement}

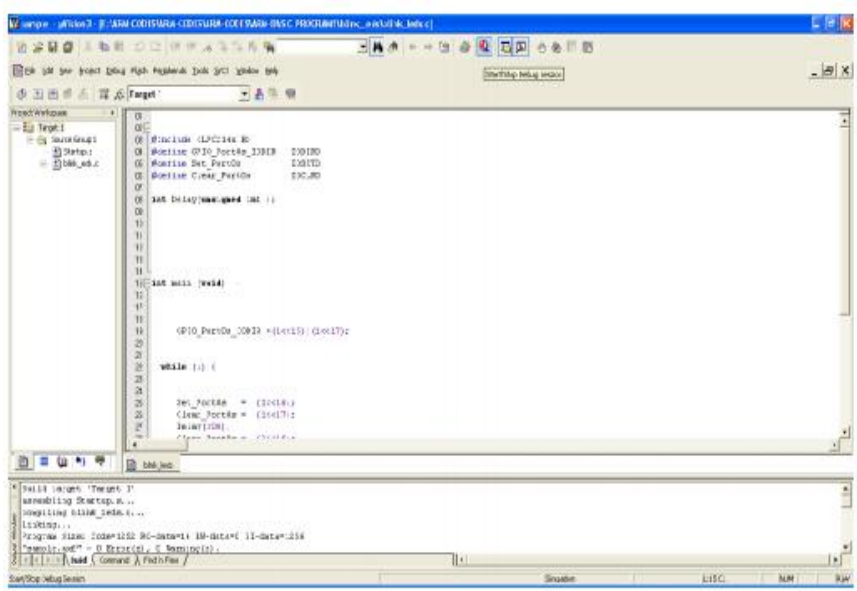

Figure 4.1Compilation of source code

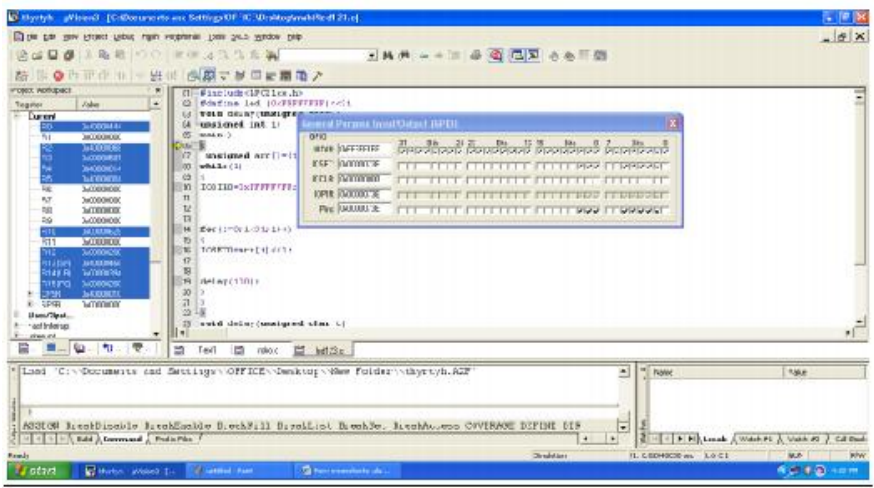

Figure 4.2 Process of Run Compilation

4.4 Flash Magic For Software Dumping Requirements

Flash Magic is a PC tool for programming flash based microcontrollers from NXP using a serial or Ethernet protocol while in the target hardware. The figures below show how the baud rate is selected for the microcontroller, how are the registers erased before the device is programmed. Browse in Hex Files and connected to LPC2148 then click ok.

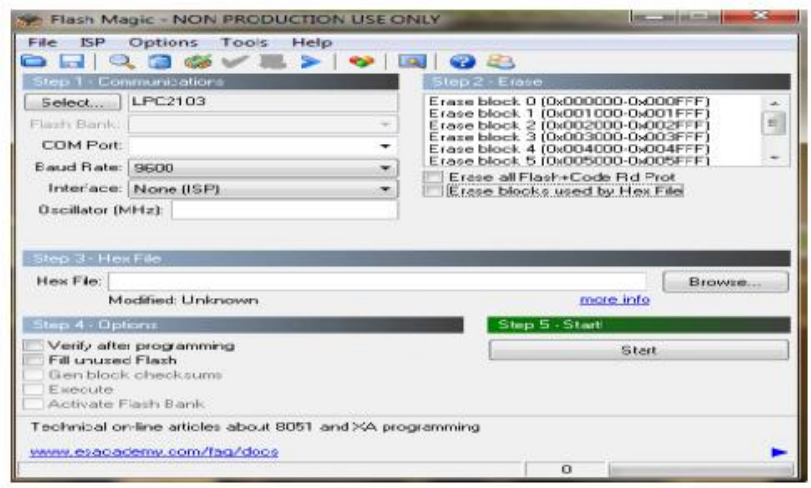

Figure 4.3 Dumping the code into micro controller

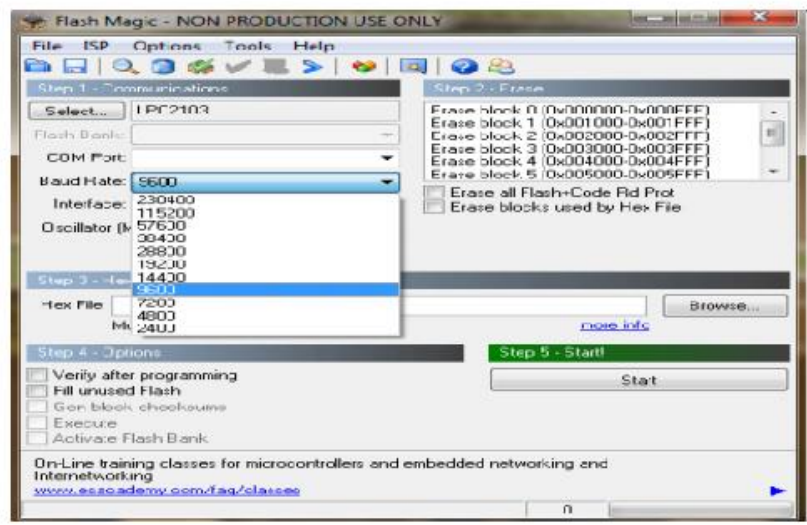

Figure 4.4 Select baud rate 9600

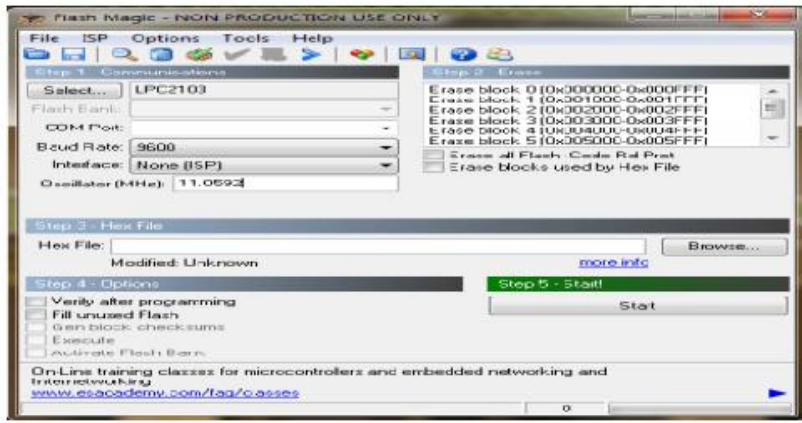

Figure 4.5 Set oscillator frequency as 11.0592 V.Results

1.The connections are made as per the circuit diagram as shown in below figure.

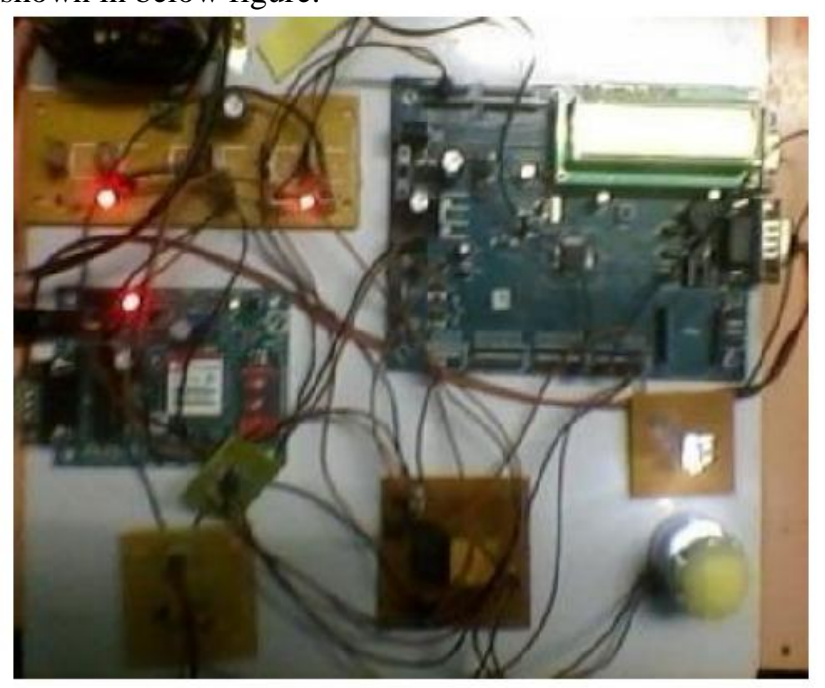

2.The GSM modem and MEMS sensors are initialized.

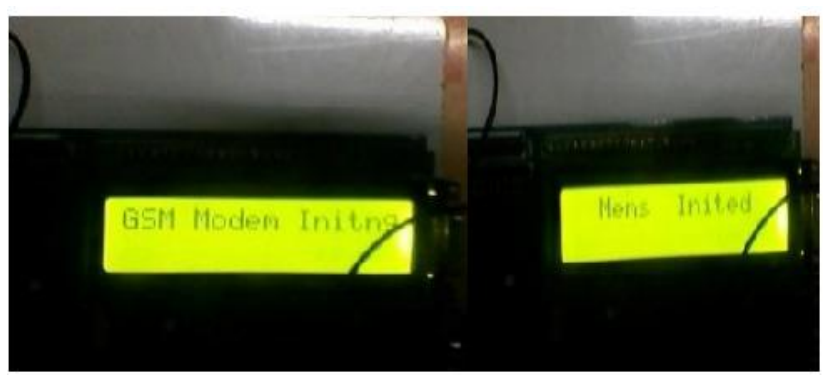

3. After initialization of GSM and MEMS Sensor it will ask a give missed call to the inserted mobile number as 
shown in below. And then giving missed call it will automatically reject the call and send a message as that your number is feeded successfully.
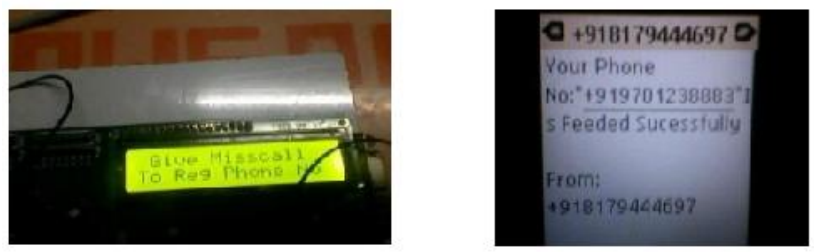

4.With the help of Sensors the GPS will decodes its latitude and longitude values.
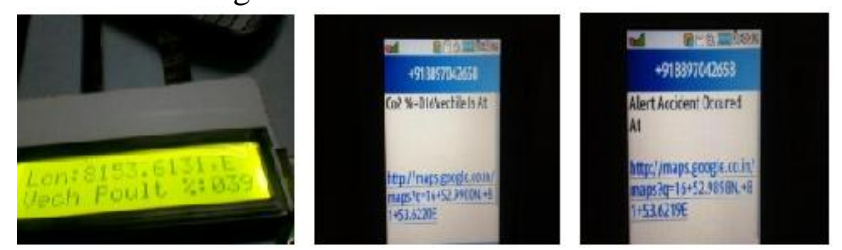

5.when the emission level beyond the threshold voltage it will send a message as shown below.

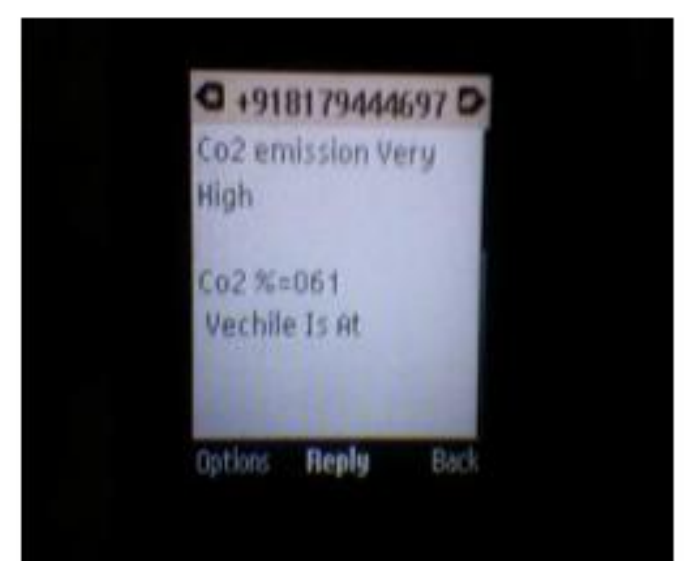

CONCLUSION

This paper consist of _-"Automated control system for Air Pollution and disaster detection in vehicles" has been successfully designed and tested. Integrating features of all the hardware components used have developed it. Presence of every module has been reasoned out and placed carefully thus contributing to the best working of the unit. Secondly, using highly advanced IC's and with the help of growing technology the project has been successfully implemented. Design is done to meet all the specifications and requirements. Software tools like Keil U vision Simulator, Preload to dump the source code into the microcontroller, Or flash magic for the schematic diagram have been used to develop the software code before realizing the hardware. It could be used as a valuable tool for real time traveler information, congestion monitoring, and system evaluation. The system can be used to quickly respond to the unexpected accidents which occur on highways or busy roads in cities.

In future it can be implemented by advanced processor. When the driver is in drunken state A Vehicle Positioning System is thus designed by using Embedded ARM controller along with GPS module. When the latitude and longitude values obtained, are fed into Google Earth software, the location of the vehicle could be found out the system will detects the driving person whether the person is drunk or not, if the person is taken alcohol, the vehicle will not start.

\section{REFERENCES}

1."The arm7 Microcontroller Architecture, Programming \&Applications - By Kenneth J Ayala.

2."The ARM Microcontroller \& Embedded Systems by Mohammed AliMazidi and Janice Gillespie Mazidi

3."Power Electronics by M D Singh and K B Khanchandan

4."Linear Integrated Circuits by D Roy Choudary\&Shail Jain

5 "Electrical Machines by S K Bhattacharya

6."ElectricalMachinesII byBLTherejaSoundarraj.V,Rajasekar.L, 7.Design of Car Black Box Based on ARM, International Journal of Microsystems Technology and Its Applications (IJMTA) Vol-1, No-2 January-2013.

8.Prof.M.Nirmala,M.Dineshkumar,-Designand Implementation of Automotive Control Features using ARM, Volume 2, Issue 5, May 2013.

9.Datasheet of LPC2148, Rev. 01 - 7 September 2005 10.P. Ajay Kumar Reddy, P.Dileep Kumar , K. Bhaskar reddy, E.Venkataramana, M.Chandra sekhar Reddy, —Black Box for Vehicles, International Journal of Engineering Inventions, Volume 1, Issue 7(October2012) PP: 06-12.

11."A Guide to the Global Positioning System (GPS) - GPS Timeline". Radio Shack. Retrieved 2010-01-14.

Referred Web Sites

1. WWW.MITEL.DATABOOK.COM

2. WWW.ATMEL.DATABOOK.COM

3. WWW.FRANKLIN.COM

4. WWW.KEIL.COM 